\title{
A Novel Blind Watermarking Scheme based on Quaternion and Joint SVD Blocks
}

\author{
Feng Liu $^{1,2, *}$ and Hao Feng ${ }^{1}$ \\ ${ }^{1}$ College of Information Engineering, Zhejiang University of Technology, \\ Hangzhou, 310014, China \\ ${ }^{2}$ Ningbo Institutes of Technology, Zhejiang University, Ningbo, 315100, China \\ lf_nit@sina.com
}

\begin{abstract}
In this paper, a novel blind color image watermarking technique using Quaternion and Joint QSVD Blocks is proposed to protect the intellectual property rights of color images. The proposed method tries to insert the watermark in the joint blocks.In this method, the color image is considered as an array of pure quaternion numbers. Then the array of pure quaternion is divided into non-overlapping blocks and performs QSVD to the blocks. The watermarking is inserted into the $S$ blocks by changing the $\sigma_{2}$ of $S$ of two adjacent blocks. Besides, in the procedure of watermark insertion and extraction, ensuring higher fidelity and robustness and resilience to several possible image attacks have been considered. The experimental results showed that the proposed method performance created watermarked images with better PSNRs and more robustness versus several attacks such as JPEG, Salt \&Pepper noise, Brightness adjustment, Sharpen and Blurring.
\end{abstract}

Keywords: Watermarking, Quaternion, QSVD, Color image

\section{Introduction}

With the development of multimedia and internet technologies, digital media security is a primary concern in the today technological world. Therefore, illegal distribution, duplication and modification of the media cause serious problems to the protection of intellectual property rights. Digital watermarking is an important and effective technique to solve the problems caused by illegal operation [1,2]. Technically, the digital watermarking aims to embed watermark data into the media without affecting its normal usage.

According to the embedding domain, the digital watermarking is classified into two classes namely spatial and transform domain. In spatial domain, the watermark data embedded operation is done by directly manipulating the pixel values of the host media. The techniques in spatial domain are less complex but poor robust against common image processing attacks. In transform domain, some techniques transform the original media to frequency domain by using DFT, DWT and DCT [3]. Then, the watermark is inserted by modifying coefficients and watermarked media is obtained by inverse transformation of the modified coefficient. Some other techniques can insert the watermark using some transformations such as SVD [9], QR decomposition and other processing methods.

According to the methods of the extraction of the watermark, the watermarking can be classified into three classifications: Firstly, non-blind watermarking which requires the original image for the watermark extraction. Secondly, semi-blind watermarking which does not require the original image for the watermark extraction, but it need the watermark and additional information. And lastly the blind watermarking that does not require the original image, watermark and additional information.

* Corresponding Author 
In the past dozen years, there are a lot of papers in gray image watermarking in literature but it is relatively rare to find some watermarking approaches which have been developed to embed a watermark into a color image. For example, S. Hongqin and L. V. Fangliang [3] presented a method that transforms the original color image from RGB to the YUV color space and scrambled by Fibonacci transformation. The authors propose the algorithm for still color image based on double scrambling technique and the wavelet transform. A robust blind method that inserts the watermark by linear interpolation in all SVD components matrixes (U, S, and V) of Red channel has been proposed in [4]. In [5], a watermarking algorithm for color image based on HSI color space and discrete wavelet transform (DWT) has been proposed. In this method, sub-blocks of intensity are divided and sorted according to the human visual system (HVS) characteristic. Then, binary watermark is embedded by modifying the DWT coefficients of low frequencies in the selected sub-blocks. [6] Suggested a watermarking technique that uses Artificial Immune Recognition System (AIRS) to protect color image intellectual property rights. The watermark is embedded in the blue channel of a color image. M-bit binary sequence embedded into the color image is used to train AIRS. Extracting the watermark is carried out using AIRS.

From the above methods, we can see that authors has been embedded the watermark information into a color channel of the color images losing color information, do not consider the watermarking algorithm on the whole without losing color information. In order to overcome the drawback, a new color image blind watermarking scheme based on quaternion and QSVD is proposed in this paper. In this proposed method, the embedded watermark can be extracted in a blind manner and the color image is represented by quaternion. In this way, we can process the host image in a holistic manner without losing color information. A series of experiments show that the proposed scheme can attain better invisibility and stronger robustness against many common processing attacks, and the comparison with the related algorithms also reveals the higher efficiency of the proposed algorithm.

The rest of the paper is organized as follows. The basic theory of the Proposed Algorithm is given in Section 2. In Section 3, the proposed watermark embedding and extraction method is described in detail. The experimental results are given and the corresponding analysis is discussed in Section 4. The conclusion is drawn in Section 5.

\section{2 .The basic Theory}

\subsection{Quaternion Representation of a Color Image}

The quaternion, which is a type of hyper complex numbers, was formally introduced by Hamilton in 1843 [8]. A quaternion consists of one real part and three imaginary parts as follows:

$$
q=a+b i+c j+d k
$$

Where $a, \mathrm{~b}, \mathrm{c}$, and $d$ are real numbers, and $i, j$, and $k$ are complex operators obeying the following rules

$$
i^{2}=j^{2}=k^{2}=-1, i j=-j i=k, j k=-k j=i, k i=-i k=j
$$

A quaternion can be regarded as the composition of a scalar part and a vector part: $q=S+V$, where $S=a, \mathrm{~V}=b i+c j+d k$.If a quaternion $q$ has a zero scalar part $(a=0)$, then $q$ is called pure quaternion, and if

The norm of $q$ is defined as

$$
\|q\|=\sqrt{a^{2}+b^{2}+c^{2}+d^{2}}
$$

Euler's formula holds for quaternion, that is $q$ has a unit norm ( $[U S V]=Q S V D(\mathrm{~A}))$, then $q$ is called unit pure quaternion. 
$e^{\mu \phi}=\cos (\phi)+\mu \sin (\phi)$

Where $\mu$ is any unit pure quaternion. We also have: $\left\|e^{\mu \phi}\right\|=1$.

In [8], Sangwine proposed to encode the three channel components of a RGB image on the three imaginary parts of a pure quaternion, that is

$f(\mathrm{x}, \mathrm{y})=f_{R}(\mathrm{x}, \mathrm{y}) \mathrm{i}+f_{G}(\mathrm{x}, \mathrm{y}) j+f_{B}(\mathrm{x}, \mathrm{y}) \mathrm{k}$

Where $f_{R}(\mathrm{x}, \mathrm{y}), f_{G}(\mathrm{x}, \mathrm{y})$ and $f_{B}(\mathrm{x}, \mathrm{y})$ are the red, green and blue components of the pixel, respectively, and ${ }^{i},{ }^{j}$ and ${ }^{k}$ are the complex operators. In recent years, quaternion has been utilized more and more in color image processing domain. Obviously, the advantage of using quaternion to represent the color image is that we can process the image in a holistic manner without losing color information.

In order to make the quaternion matrix more convenient to operate, each component of the pixel was normalized, that is

$$
f(\mathrm{x}, \mathrm{y})=\left(f_{R}(\mathrm{x}, \mathrm{y}) \mathrm{i}+f_{G}(\mathrm{x}, \mathrm{y}) j+f_{B}(\mathrm{x}, \mathrm{y}) \mathrm{k}\right) / 256
$$

Figure 1 illustrates the details of using quaternion to represent the color image.

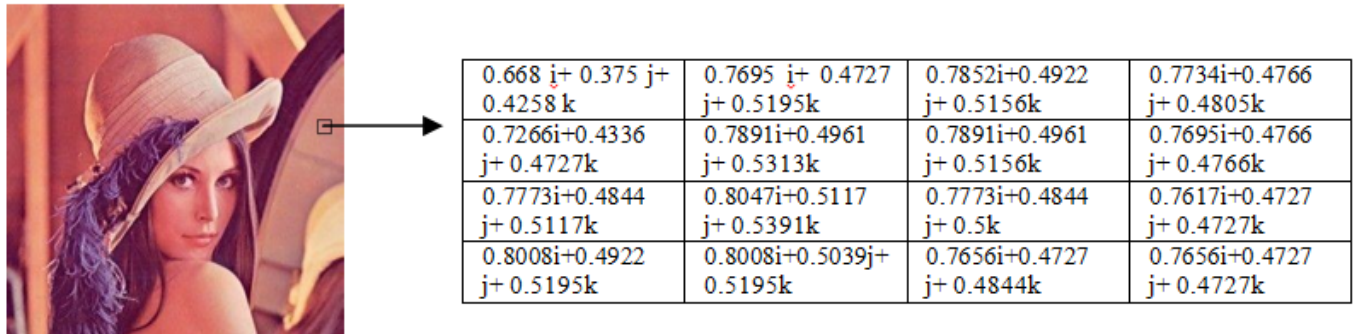

Figure 1. The Details of using Quaternion to Represent the Color Image $(4 \times 4)$

\subsection{QSVD of Color Image}

Supposing $A$, the quaternion matrix singular value decomposition (QSVD) of $A$ can be represented as follows [10]:

$A=U$ diag $\left(\sigma_{1}, \sigma_{2}, \mathrm{~L}, \sigma_{n}\right) \mathrm{V}$

Where QSVD(A), $\sigma_{1} \geq \sigma_{2} \geq \mathrm{L} \geq \sigma_{n} \geq 0$ are $n$ eigenvalues of $A$, (7) can be simply represented by

$\left[\begin{array}{lll}U & \mathrm{~S} & \mathrm{~V}\end{array}\right]=\mathrm{QSVD}(\mathrm{A})$

According to (5), a color image can be represented by the quaternion matrix. A color image with $\mathrm{m}$ rows and $\mathrm{n}$ columns can be represented by

$$
A=\left(q_{i j}\right)_{m \times n}, q_{i j} \in Q
$$

The quaternion theory introduces many new methods and theory into color image processing. These methods treat the three channels of a color image as an integer to handle, so the intrinsic mapping relational ship between three channels can be maintained. In order to embed watermark information to the image, the color image is transferred by QSVD,

$$
[U S V]=Q S V D(\mathrm{~A})
$$

Where $s$ is a diagonal matrix, and it can be written as

For example:

$$
S=\operatorname{diag}\left(\sigma_{1}, \sigma_{2}, \mathrm{~L}, \sigma_{n}\right)
$$




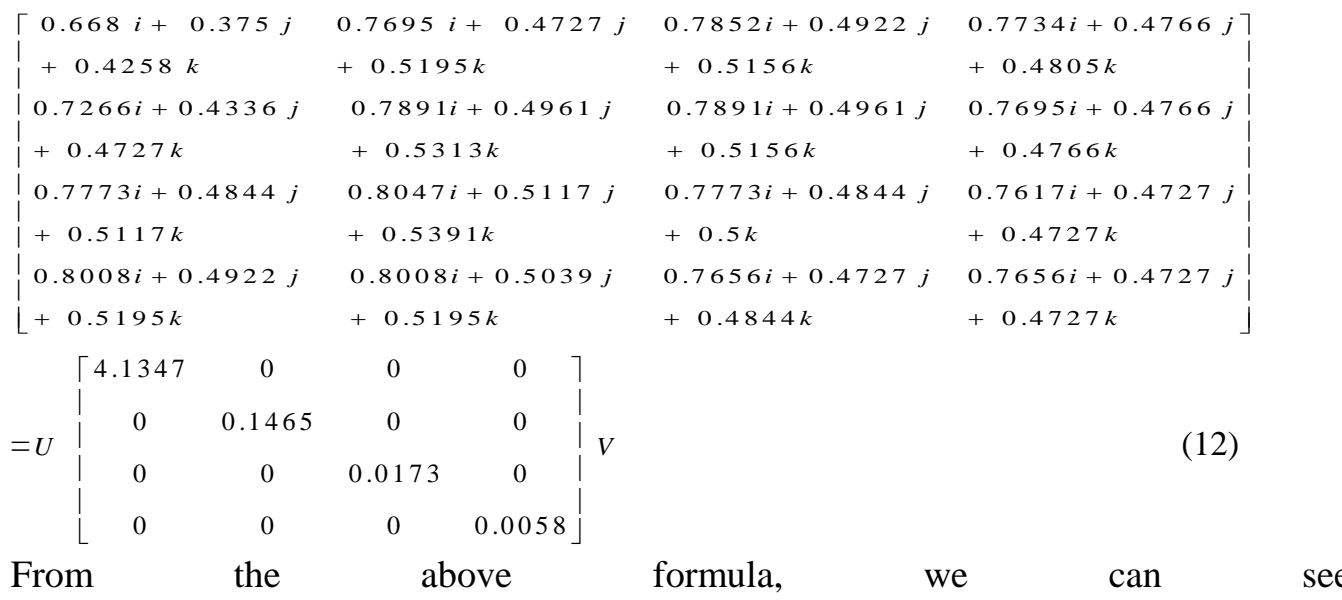
that $\sigma_{1}=4.1347, \sigma_{2}=0.1465, \sigma_{3}=0.0173, \sigma_{4}=0.0058$,

$\sigma_{1}>\sigma_{2}>\sigma_{3}>\sigma_{4}$, in order to visualize the impact of the $\sigma_{i}$, a series of experiments has been done : firstly, we set the all eigenvalues of $s$ to remain unchanged besides $\sigma_{i}$, then a new matrix is formed and it can be written as $S^{\prime}=\operatorname{diag}\left(\sigma_{1}, \sigma_{2}, \sigma_{i}^{\prime}, \mathrm{L}, \sigma_{n}\right)$. The inverse QSVD transformation is employed to get a color image. The recovered image with the $\sigma_{i}$ of $S$ being changed with different degree are presented in Table 1.

Table 1. The Recovered Image with the $\sigma_{i}$ of $s$ Being Changed

\begin{tabular}{|c|c|c|c|}
\hline & $\sigma_{i}=0.5 \sigma_{i}$ & $\sigma_{i}=1.5 \sigma_{i}$ & $\sigma_{i}=2.5 \sigma_{i}$ \\
\hline \multicolumn{4}{|l|}{$\begin{array}{l}\text { Image } \\
\text { with } \sigma_{1} \\
\text { being } \\
\text { changed }\end{array}$} \\
\hline$P S N R_{1}$ & 11.1292 & 13.8236 & 7.9273 \\
\hline \multicolumn{4}{|l|}{$\begin{array}{l}\text { Image } \\
\text { with } \sigma_{2} \\
\text { being } \\
\text { changed }\end{array}$} \\
\hline$P S N R_{2}$ & 37.4672 & 37.9582 & 29.0986 \\
\hline \multicolumn{4}{|l|}{$\begin{array}{l}\text { Image with } \\
\sigma_{3} \text { being } \\
\text { changed }\end{array}$} \\
\hline$P S R_{3}$ & 44.3600 & 44.4722 & 36.1317 \\
\hline
\end{tabular}




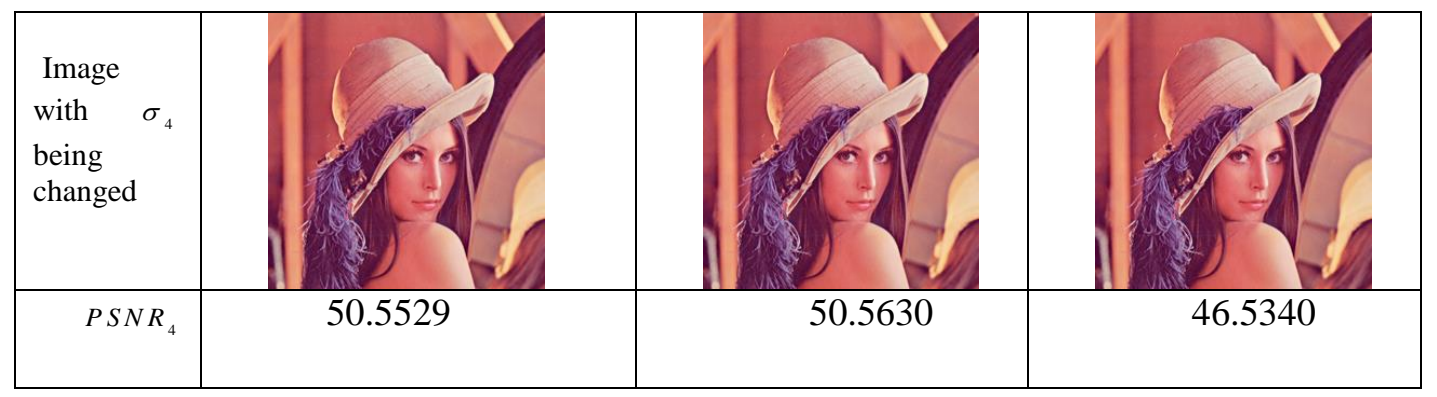

In light of Table 1, we can see that in the same degree that the $\sigma_{i}$ being changed, $P S N R_{4}<P S N R_{3}<P S N R_{2}<P S N R_{1}$, which means that the larger the value of coefficient, the greater the effect of the coefficient on image quality. In addition, any alteration to the largest coefficients of the $S$ matrices of an image leads to significant distortions in image quality. In order to achieve a balance between transparency and robustness, we should insert the watermark information to the original image by changing the $\sigma_{2}$ of $s$.

\section{Our Proposed Algorithm}

This section describes our proposed novel blind color image watermarking technique using Quaternion and Joint QSVD Blocks is to protect the intellectual property rights of color images, which insert the watermarking information into the $S$ blocks by changing the $\sigma_{2}$ of $S$ of two adjacent blocks. The two procedures are described in Subsections 3.1 and 3.2, respectively.

\subsection{Embedding Procedure}

In order to use the characteristics of the QSVD domain to embed a watermark into a host image, we explored the coefficients of $s$ matrix in each block as the Table 1 represents. Based on our observation, in the same degree that the $\sigma_{i}$ being changed, the larger the value of coefficient, the greater the effect of the coefficient on image quality. An overview of the embedding procedure is shown in Figure 1. The size of host color image $I$ represented by quaternion is $m \times n$.The host image is divided into nonoverlapping

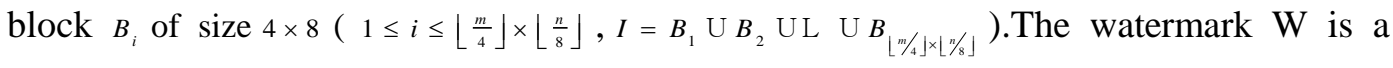
binary image of size $u \times v$ bits $\left(\mathrm{W}=\left(\mathrm{w}_{1}, \mathrm{w}_{2}, \mathrm{~L}, \mathrm{w}_{\left\lfloor\frac{m}{4} \times \times \frac{n}{l}\right]}\right)\right.$ and $\left.\mathrm{w}_{1} \in\{1,0\}\right)$.The block $B_{i}$ is divided into two nonoverlapping blocks $B_{i 1}$ and $B_{i 2}$ of size $4 \times 4$, then Apply QSVD to $B_{i 1}$ and $B_{i 2}$ and get $\left[\mathrm{U}_{i 1}, S_{i 1}, V_{i 1}\right]$ and $\left[\mathrm{U}_{i 2}, S_{i 2}, V_{i 2}\right]$. In order to embed the watermark to the host color image, we change the value of the $\sigma_{2}$ in the matrices $S_{i 1}$ and $S_{i 2}$ according to the value of the pixel of the watermark bit. The changing procedure of the $\sigma_{2}$ in the matrices $S_{i 1}$ and $S_{i 2}$ is as follows.

If the watermark bit is " 1 ", $S_{i 2}(2,2)=\alpha * S_{i 1}(2,2)$; if the watermark bit is " 0 ", $S_{i 1}(2,2)=\alpha * S_{i 2}(2,2)$. According to the Table $1,1 \leq \alpha \leq 3$.

The embedding algorithm in detail is as following a few steps:

Step 1: Using the normalized quaternion to represent the host color image $I$.

Step 2: Divide the host color image into nonoverlapping blocks $B_{i}$ of size $4 \times 8$ 


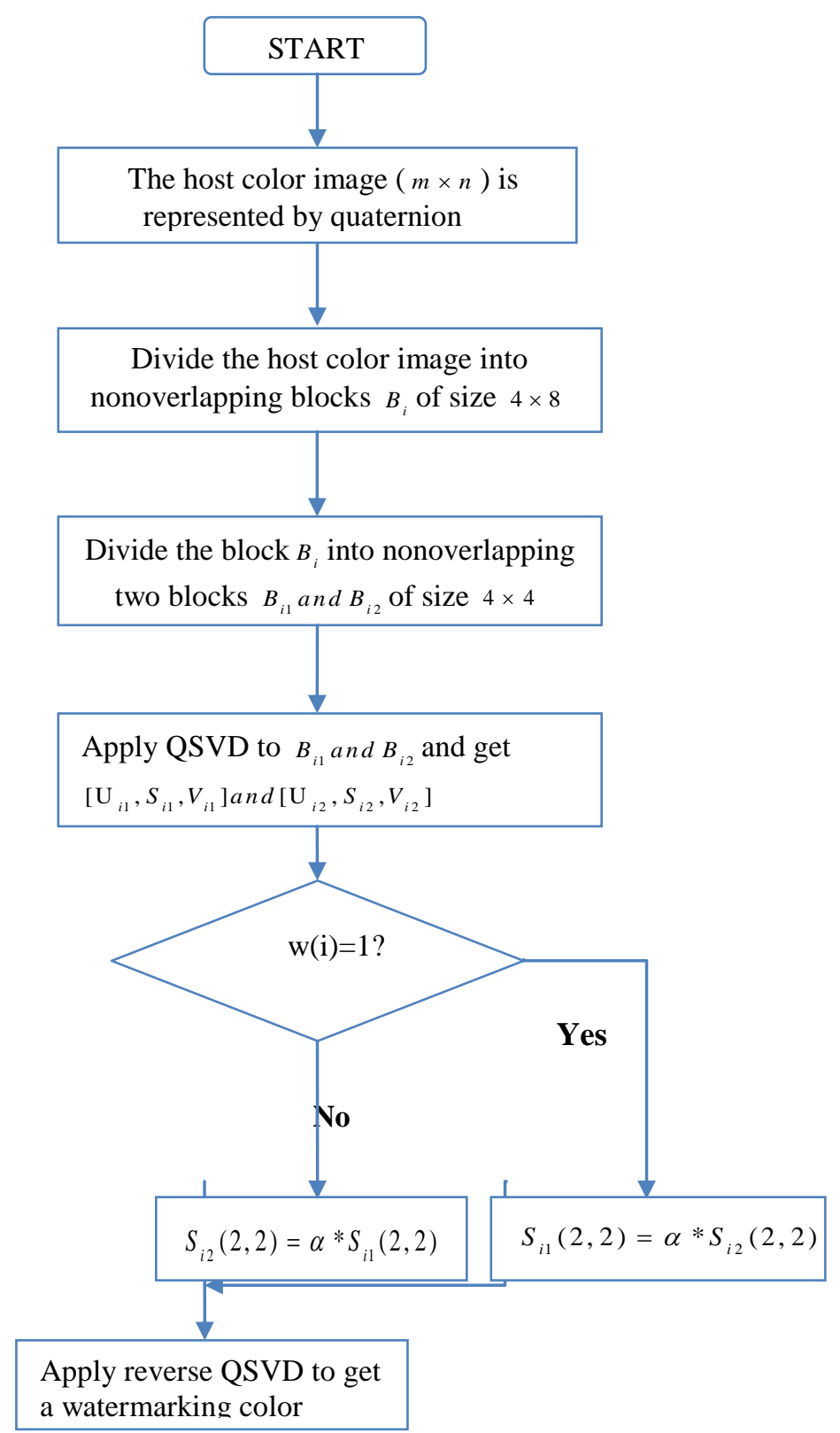

Figure 1. Flowchart of Embedding Procedure

Step 3: Divide the block $B_{i}$ into nonoverlapping two blocks $B_{i 1}$ and $B_{i 2}$ of size of $4 \times 4$.

Step 4: Apply QSVD to $B_{i 1}$ and $B_{i 2}$ and get $\left[\mathrm{U}_{i 1}, S_{i 1}, V_{i 1}\right]$ and $\left[\mathrm{U}_{i 2}, S_{i 2}, V_{i 2}\right]$

Step 5: Embedding the watermark information to the $s_{i 1}$ and $s_{i 2}$ on the basis of the value of watermark bit. If the watermark bit is " 1 ",,$S_{i 2}(2,2)=\alpha * S_{i 1}(2,2)$; if the watermark bit is " 0 ", $S_{i 1}(2,2)=\alpha * S_{i 2}(2,2) .1 \leq \alpha \leq 3$.

Step 6: Apply reverse QSVD to get a watermarking color image $I^{*}$ 


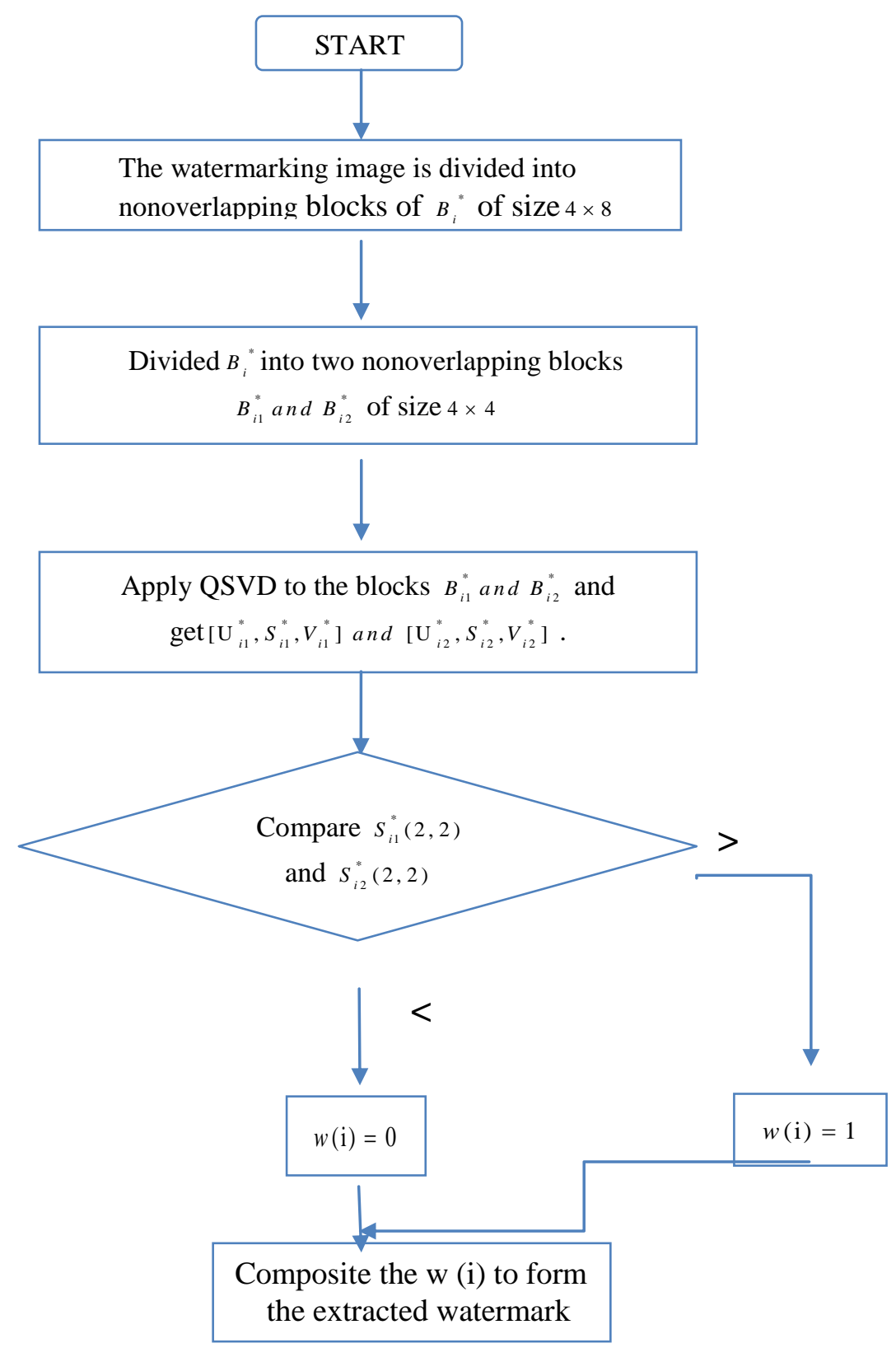

Figure 2. Flowchart of Extracting Procedure

\subsection{Extracting Procedure}

In this paper, we propose a blind watermarking algorithm that the watermark information is extracted without the original host image and the original watermark information. Figure 2 presents a flowchart of our extracting procedure. The watermarking image is first divided into nonoverlapping blocks of $B_{i}{ }^{*}$ of size $4 \times 8$, then the $B_{i}{ }^{*}$ is divided into two nonoverlapping blocks $B_{i 1}^{*}$ and $B_{i 2}^{*}$ of size $4 \times 4$. Apply QSVD to the blocks $B_{i 1}^{*}$ and $B_{i 2}^{*}$ and get $\left[\mathrm{U}_{i 1}^{*}, S_{i 1}^{*}, V_{i 1}^{*}\right]$ and $\left[\mathrm{U}_{i 2}^{*}, S_{i 2}^{*}, V_{i 2}^{*}\right]$.Subsequently, in accordance with the comparison results of $s_{i 1}^{*}(2,2)$ and $s_{i 2}^{*}(2,2)$, we can get the value of the watermark bit .The criterion of achieve the value of the watermark bit is as following: If $s_{i 1}^{*}(2,2)>$ $s_{i 2}^{*}(2,2), \mathrm{w}(\mathrm{i})=1$; If $s_{i 1}^{*}(2,2)<s_{i 2}^{*}(2,2), \mathrm{w}(\mathrm{i})=0$. At last, composite the $\mathrm{w}$ (i) to form the extracted watermark. 
The Extracting algorithm in detail is as following a few steps:

Step 1: The watermarking color image $I^{*}$ is divided into nonoverlapping blocks of $B_{i}$ of size $4 \times 8$

Step 2: Divided $B_{i}{ }^{*}$ into two nonoverlapping blocks $B_{i 1}^{*}$ and $B_{i 2}^{*}$ of size $4 \times 4$

Step 3: Apply QSVD to the blocks $B_{i 1}^{*}$ and $B_{i 2}^{*}$ and get $\left[\mathrm{U}_{i 1}^{*}, S_{i 1}^{*}, V_{i 1}^{*}\right]$ and $\left[\mathrm{U}_{i 2}^{*}, S_{i 2}^{*}, V_{i 2}^{*}\right]$.

Step 4: Compare $s_{i 1}^{*}(2,2)$ and $s_{i 2}^{*}(2,2)$, If $s_{i 1}^{*}(2,2)>s_{i 2}^{*}(2,2), \mathrm{w}(\mathrm{i})=1$; If $s_{i 1}^{*}(2,2)<$ $S_{i 2}^{*}(2,2), \mathrm{w}(\mathrm{i})=0$

Step 5: Composite the w (i) to form the extracted watermark

\section{Experiment Results and Analysis}

This section is divided into three subsections. The first will introduce the evaluation criteria. The second subsection will introduce the visual result of the proposed algorithms. Third subsection will compare the algorithm with other existing algorithms. A set of experiments was developed in the MATLAB R2010b environment, while all measurements are performed on a Lenovo laptop with a $2.1 \mathrm{GHz}$ Intel Pentium processor and $4 \mathrm{~GB}$ of RAM.A number of common benchmark RGB images with $256 \times 256$ pixels size are applied for the experiments, while the binary watermark Logo sized as $64 \times 32$ bits (2048bits in total) is used as the watermark information (Figure 3)

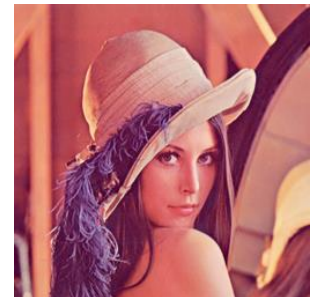

(a)

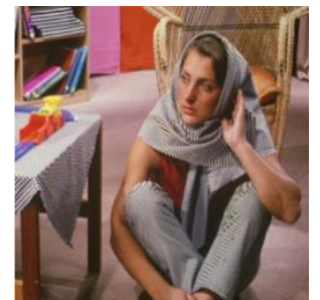

(b)

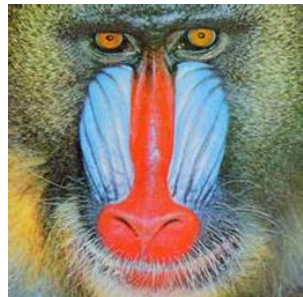

(c)
ZJUNIT

(d)

Figure 3. The Benchmark Images (a) Lena, (b) Barbara, (c) Mandrill And (d) The Binary Watermark Logo

\subsection{Evaluation Criteria}

In general, there are two measures; $P S N R$ (peak signal-to-noise) and $B C R$ (bit correction ratio) were used to evaluate the performance of our proposed scheme. The PSNR value, which is defined in Equation (12), was used to measure the image quality of the watermarked and original images.

$$
P S N R=10 \log _{10} \frac{255^{2}}{M S E} d B
$$

Where 255 represents the maximum pixel value of a gray-level image, and the mean square error ( $M S E$ ) of an image is depicted in Equation (13).

$$
M S E=\frac{\sum_{r}^{h t} \sum_{s}^{w d}\left(\mathrm{x}_{r s}-\mathrm{x}_{r s}^{\prime}\right)^{2}}{H \times W}
$$

Here, the notations $h t$ and $w d$ represent the height and width of an image, respectively.

If $P S N R$ is used to measure the image quality of the watermarked image, $x_{r s}$ is the pixel value of the position $(r, s)$ in an original image and $x_{r s}^{\prime}$ is the pixel value of the watermarked image. Generally, the higher the $P S N R$ value of a watermarked image is, the better the image quality will be. Furthermore, the $B C R$ defined in Equation (14) is used to measure the correction ratio of the extracted watermark. 


$$
B C R=\frac{\sum_{i=1}^{n \times n} \overline{w_{i} \oplus w_{i}^{\prime}}}{n \times n} \times 100 \%
$$

Where $w_{i}$ and $w_{i}$ are the $i$ th binary value of the original watermark and of the extracted watermark, respectively, and $\oplus$ indicates an exclusive-OR operator. Note that a higher $B C R$ implies greater similarity between the original watermark and the extracted watermark.

\subsection{The Experiment Results without Being Attacked}

The experiment results of the proposed watermark embedding algorithm without being attacked are shown in Table 2. From the Table 2, we can see that the watermark is imperceptible and there are no visible differences between the two by comparing the original image signal with the image signal after embedding the watermark into the original image.

Table 2. The Experiment Results without Being Attacked

\begin{tabular}{|c|c|c|c|}
\hline $\begin{array}{l}\text { The original } \\
\text { color image }\end{array}$ & Lena & Barbara & Mandrill \\
\hline $\begin{array}{c}\text { The } \\
\text { watermarked } \\
\text { color image }\end{array}$
\end{tabular}

\subsection{The Experiment Results with Being Attacked}

In this section, a set of experiments were conducted to attack the watermarked color image in order to evaluate the proposed algorithm. The attacking conditions simulated for the algorithm's evaluation consisting of common signal processing: JPEG(95\%,85\%),Salt \&Pepper(0.002,0.005,0.01),Brightness adjustment(light, dark), Sharpen and Blurring.

Table 3 shows us that the performance of the proposed scheme in image and BCR under Salt \&Pepper noise in different degree. We can see that even thouth the watermarked image is attacked by Salt \&Pepper noise with a factor of 0.01 , the BCRs of the extracted watermark is still $85.84 \%$ 


\section{Table 3. The Experiment Results Being Attacked by Sal t\& Pepper Noise}

\begin{tabular}{|c|c|c|c|}
\hline & $\begin{array}{l}\text { Salt \& Pepper } \\
0.002\end{array}$ & $\begin{array}{c}\text { Salt \& Pepper } \\
0.005\end{array}$ & $\begin{array}{c}\text { Sal t\& Pepper } \\
0.01\end{array}$ \\
\hline \multicolumn{4}{|l|}{$\begin{array}{c}\text { The } \\
\text { watermarked } \\
\text { color image }\end{array}$} \\
\hline \multicolumn{4}{|l|}{$\begin{array}{c}\text { The } \\
\text { watermarked } \\
\text { color image } \\
\text { with being } \\
\text { attacked }\end{array}$} \\
\hline$P S N R$ & 30.8645 & 27.5693 & 24.8593 \\
\hline $\begin{array}{l}\text { The watermark } \\
\text { Being extracted }\end{array}$ & $7 f(x)$ & 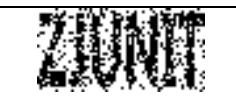 & 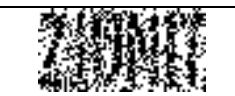 \\
\hline$B C R$ & $96.34 \%$ & $91.21 \%$ & $85.84 \%$ \\
\hline
\end{tabular}

From Table 4, we can see that with being attacked by brightness adjustment and sharpen; our proposed algorithm shows superior performance. Especially, the PSNR of the tampered image is only $12.0869 \mathrm{~dB}$, but the BCR of the extracted watermark is still $100 \%$.It is clear that the watermarks have been fully extracted. In other words, the difference between the extracted watermark and the original image is zero.

\section{Table 4. The Experiment Results Being Attacked by Brightness Adjustment and Sharpen}

\begin{tabular}{|c|c|c|c|}
\hline $\begin{array}{c}\text { The watermarked } \\
\text { color image } \\
\text { adjustment } \\
\text { (light) }\end{array}$ & $\begin{array}{c}\text { Brightness } \\
\text { Adjustment } \\
\text { (dark) }\end{array}$ \\
\hline $\begin{array}{c}\text { The watermarked } \\
\text { color image } \\
\text { with being } \\
\text { attacked }\end{array}$
\end{tabular}

There are a set of tests will investigate the performance of the proposed algorithm attacked under blurring and Jpeg compression. In Table 5, we can see that the PSNR of the tampered image attacked by blurring is $34.2462 \mathrm{~dB}$, the BCR of the extracted 
watermark is $97.75 \%$.The compression ratios used are $0.95,0.85$, the PSNR of the noisy color image are 30.5926 and 29.9999 respectively, the BCR of the extracted watermark is 98.58\% and $94.73 \%$ respectively which indicate that our method has the ability to resist attacks of blurring and Jpeg compression.

Table 5. The Experiment Results Being Attacked by Blurring and Jpeg

$\left.\begin{array}{|c|c|c|c|}\hline \begin{array}{c}\text { The } \\ \text { watermarked } \\ \text { color image }\end{array} & \text { Blurring } & \operatorname{Jpeg}(95) \\ \hline \begin{array}{c}\text { The } \\ \text { watermarked } \\ \text { color image } \\ \text { with being } \\ \text { attacked }\end{array} \\ \hline P S N R\end{array}\right)$

Table 6. Performance Comparisons between Other Watermark Schemes and Ours

\begin{tabular}{|l|l|l|l|l|}
\hline $\begin{array}{l}\text { Performance } \\
\text { comparisons }\end{array}$ & \multicolumn{1}{|c|}{$\begin{array}{l}\text { Chandra } \\
{[11]}\end{array}$} & $\begin{array}{l}\text { Bao and Ma } \\
{[4]}\end{array}$ & $\begin{array}{l}\text { Hwang et al. } \\
{[12]}\end{array}$ & Ours scheme \\
\hline $\begin{array}{l}\text { Processing } \\
\text { domain }\end{array}$ & SVD domain & $\begin{array}{l}\text { Wavelet and } \\
\text { SVD domains }\end{array}$ & Spatial domain & QSVD domain \\
\hline $\begin{array}{l}\text { Blind } \\
\text { watermarking } \\
\text { method }\end{array}$ & No & No & Yes & Yes \\
\hline Robustness & High & High & Low & High \\
\hline $\begin{array}{l}\text { Embedding } \\
\text { quality }\end{array}$ & High & General High & General High & General High \\
\hline $\begin{array}{l}\text { Extra data is } \\
\text { required for } \\
\text { watermark } \\
\text { extraction }\end{array}$ & $\begin{array}{l}\text { The original } \\
\text { image and } \\
\text { original } \\
\text { watermark }\end{array}$ & $\begin{array}{l}\text { Quantization } \\
\text { factors }\end{array}$ & No & No \\
\hline
\end{tabular}

By comparing the performance of three similar watermark schemes with that of our proposed scheme in Table 6, neither extra data nor the original host image is required during the extracted procedure in our proposed scheme. Hwang et al.'s scheme [12] can provide high image quality and low robustness, which their scheme cannot work well after the watermarked images are attacked by other image processing attacks. With regard to Chandra's scheme [11], it requires both the original watermark and the original image during the watermark extraction procedure. Bao and Ma's scheme [4] successfully uses the quantization parameters to enhance the image quality of the watermarked image, but the quantization parameters must be stored for later watermark extraction. 


\section{Conclusions}

This paper presents a novel blind color image watermarking technique using Quaternion and Joint QSVD Blocks to protect the intellectual property rights of color images. The proposed method tries to insert the watermark in the joint blocks .In this method, the color image is considered as an array of pure quaternion numbers. Then the array of pure quaternion is divided into non-overlapping blocks and performs QSVD to the blocks. The watermarking is inserted into the $\mathrm{S}$ blocks by changing the $\sigma_{2}$ of $S$ of two adjacent blocks. Besides, in the procedure of watermark insertion and extraction, ensuring higher fidelity and robustness and resilience to several possible image attacks have been considered. The experimental results showed that the proposed method performance created watermarked images with better PSNRs and more robustness versus several attacks such as JPEG, Salt \&Pepper noise, Brightness adjustment, Sharpen and Blurring.

\section{References}

[1] B. Sebastiano, E. Sabu U, Adrian and W. Marcel, "Multimedia in forensics, security, and intelligence", IEEE Multi Media, vol. 19, no. 1, (2012), pp. 17-19.

[2] P. Lin, J. Lee and C. Chang, "Protecting the content integrity of digital imagery with fidelity preservation", ACM Trans. Multim. Comput., Commun., Appl., vol. 7, no. 3, (2011), pp. 1-15.

[3] S. Hongqin and L. V. Fangliang, "A blind watermark algorithm for color image based on dual scrambling technique", In Third International Symposium on Intelligent Information Technology and Security Informatics (IITSI), (2010) April 2-4, pp. 781-785.

[4] A. Benhocine, L. Laouamer, L. Nana and A. C. Pascu, "New images watermarking scheme based on singular value decomposition”, J. Inform. Hiding Multimedia Signal Process, vol. 4, (2013) January 1, pp. 9-18.

[5] F. Kong and Y. Peng, "Color image watermarking algorithm based on HSI color space", in: 2nd International Conference on Industrial and Information Systems (IIS), vol. 2, (2010) July 10-11, pp. 464-467.

[6] O. Findık, B. Lu and E. Ulker, "A color image watermarking scheme based on artificial immune recognition system”, Expert Syst. Appl., vol. 38, no. 3, (2010), pp. 1942-1946.

[7] I. L. Kantor and A. S. Solodovnikov, "Hyper complex Number", An Elementary Introduction to Algebras, Springer-Verlag, New York, (1989).

[8] S. J. Sangwine, "Fourier transforms of color images using quaternion or hyper complex numbers, Electron", Lett., vol. 32, (1996), pp. 1979-1980.

[9] A. Benhocine, L. Laouamer, L. Nana and A. C. Pascu, "New images watermarking scheme based on singular value decomposition”, J. Inform. Hiding Multimedia Signal Process, vol. 4, (2013) January 1, pp. 9-18.

[10] L. Yanshan, “A New Color Image Blind Watermarking Algorithm Based on Quaternion”, in: ICSP2010 Proceedings, (2010), pp. 1698-1701.

[11] D. V. S. Chandra, "Digital image watermarking using singular value decomposition", Proc. of The45th Midwest Symposium on Circuits and Systems (MWSCAS 2002), vol. 3, (2002), pp. 264-267.

[12] M. S. Hwang, C. C. Chang and K. F. Hwang, "A watermarking technique based on one-way hash functions”, IEEE Trans. Consumer Electronics, vol. 45, no. 2, (1999), pp. 286-294.

\section{Authors}

Feng Liu, is currently a Ph.D. student in the College of Information Engineer, Zhejiang University of Technology in China. His research focuses on image processing, multimedia watermarking and information forensics and he has published several papers in scholarly journals and international conferences in the above research areas.

Hao Feng, is currently a professor in the College of Information Engineer, Zhejiang University of Technology in China. Professor Feng has published over 60 research papers in scholarly journals and international conferences. His main research interests include pattern recognition, artificial neural network and image processing. 